(di) https://doi.org/10.37843/rted.v9i2.147

\title{
La Enseñanza Tecnoemocional en la Educación del Siglo XXI
}

\section{Technoemotional Teaching in the Education of the XXI Century}

Ruth M. Mujica-Sequera ${ }^{1}$

\section{อ}

\section{EDICIÓN: 曲:eIVTAC}

Recibido: 8/julio/2020

Aceptado: 26/agosto/2020

Publicado: 25/septiembre/2020

${ }^{1}$ Omán

\section{IIIIInstitución}

${ }^{1}$ Grupo Docentes 2.0 C.A.

\section{Correo Eletrónico}

1ruth.mujica@docentes20.com

\section{(iD) ORCID}

${ }^{1}$ https://orcid.org/0000-0002-2602-5199

\section{Citar así: C4APA / IEEE}

Mujica-Sequera, R. (2020). La Enseñanza Tecnoemocional en la Educación del Siglo XXI. Revista Tecnológica-Educativa Docentes 2.0, 9(2), 71-78. https://doi.org/10.37843/rted.v9i2.147

R. Mujica-Sequera, "La Enseñanza Tecnoemocional en la Educación del Siglo XXI", RTED, vol. 9, n. ${ }^{\circ}$ 2, pp.71-78, sep. 2020.

\section{Resumen}

Hoy en día, la sociedad demanda que los docentes tengan competencias y estén alfabetizados digitalmente para poder ejercer una práctica pedagógica que admita el aprendizaje significativo de los estudiantes del siglo XXI. La sociedad actual ha tenido una fuerte integración de la tecnología en todos los ámbitos de la vida, lo que ha permitido que el hombre tenga acceso a una extensa cantidad de información desde cualquier parte del mundo. Las generaciones del siglo XXI están bajo la influencia tecnología, lo cual implica que los docentes de formación del siglo $\mathrm{XX}$ han tenido que desaprender para poder enriquecer su práctica pedagógica apoya en tecnología. El presente estudio surgió de la necesidad de crear aulas que apoyen las necesidades de los estudiantes, entre ello, la motivación para aprender a aprender, como, los efectos que la tecnología tiene sobre ellos. Fue una investigación de corte metodológico cuantitativo, el propósito de este fue revelar la realidad social desde una perspectiva objetiva para poder así crear planes pedagógicos para mejorar la instrucción Tecnológica-Educativa. La investigación fue consumada en la plataforma Tecnológica-Educativa Docentes 2.0, con una población de 369 estudiantesdocentes. Se realizó encuestas para evaluar el conocimiento y motivación de la población objeto de estudio, igualmente, la revisión de la planificación del aula. Por ende, los resultados revelaron que los estudiantes-docentes se sentían motivados por el uso de la tecnología en el aula, lo cual, impulsa al fortalecimiento del docente a nivel mundial apoyado por las Tecnologías del Aprendizaje y del Conocimiento (TAC).

Palabras clave: Tecnología, educación, TAC, enseñanza, tecnoemocional.

\section{Abstract}

Today, society demands that teachers have skills and be digitally literate to exercise a pedagogical practice that supports meaningful learning for 21 st century students. Today's society has had a strong integration of technology in all areas of life, which has allowed man to have access to an extensive amount of information from anywhere in the world. The generations of the 21 st century is under the influence of technology, which implies that the training teachers of the 20th century have had to unlearn to enrich their pedagogical practice supported by technology. The present study arose from the need to create classrooms that support the needs of the students, among them, the motivation to learn to learn, such as the effects that technology has on them. It was a quantitative methodological research, the purpose of this was to explain social reality from an objective perspective to create pedagogical plans to improve Technological-Educational instruction. The research was completed on the Technological-Educational Docentes 2.0 platform, with a population of 369 student-teachers. Surveys were carried out to evaluate the knowledge and motivation of the population under study, as well as the revision of classroom planning. Therefore, the results revealed that student-teachers were motivated using technology in the classroom, which drives the strengthening of teachers worldwide supported by Learning and Knowledge Technologies (TAC).

Keywords: Knowledge management process, sociocultural context / reality, academic context / reality, innovation, revitalization. 


\section{Introducción}

Hoy en día, la sociedad demanda que los docentes tengan competencias y estén alfabetizados digitalmente para poder ejercer una práctica pedagógica que admita el aprendizaje significativo de los estudiantes del siglo XXI. El autor Mujica-Sequera (2018) sustenta en el blog Docentes 2.0 que el alfabetismo digital "aparecido como una condición para reducir la brecha digital". Los educandos del siglo XXI están inmersos en la tecnología con acceso a una extensa cantidad de información desde cualquier parte del mundo. En el ámbito educativo son muchos los estudios que aseveran la integración tecnológica porque es beneficiosa, significativa e imprescindible para que las instituciones educativas se desempeñen exitosamente. No obstante, muchos docentes están resistentes al cambio porque en mucho de los casos les ocasiona tecnoestrés, lo cual es sustentado como "la experiencia de estrés derivado de la introducción de las TIC en el trabajo" (Mujica-Sequera, 2020).

En el año 2013, se realizó una encuesta en la plataforma Tecnológica-Educativa Docentes 2.0 y se obtuvo que el $92 \%$ de los estudiantes-docentes poseían cierto tipo de tecnología y acceso a internet en sus hogares, pero menos de la mitad de los encuestados no aprovechaban esa tecnología para las asignaciones escolares. Lo que reveló que el paradigma didáctico de la formación con tecnología presentaba elementos que perjudicaban la motivación de los educandos a la hora de desarrollar sus actividades, debido a que los educadores no incorporaban o utilizaban técnicas y metodologías tecnológicas a nivel instruccional y motivacional en el quehacer didáctico-pedagógico. Por lo tanto, fue considerado que "prevenir o reducir la frustración del estudiante es de gran importancia" (Mujica-Sequera, 2017) para la educación del siglo XXI.

Como todas las personas conocen, no todos los individuos aprenden de la misma manera, por ello, es importante que los docentes conozcan ¿Cuantos tipos de aprendizaje existe? porque esto les permitirá enriquecer su quehacer pedagógico y la didáctica reflejará el valor intrínseco del proceso de enseñanzaaprendizaje significativo, potenciado por el interés y la motivación de todos los involucrados. Por ende, la educación apoyada en tecnología, tal como lo sustenta UNESCO (1998) "apoyada por las TIC (tecnologías, redes de telecomunicaciones, videoconferencias, TV digital, materiales multimedia)" (p. 56) potencian los espacios educativos e-learning.

No obstante, para ello, se debe tener presente que "no se puede enseñar de acuerdo con nuestro tipo de aprendizaje. Se debe evaluar a los estudiantes antes de programar un aula virtual" (Mujica-Sequera, 2020), en consecuencia, como, lo señala Barráez (2020) "la consideración de cómo enseñamos y aprendemos en las universidades está directamente relacionada con el nivel de formación profesional de nuestros facilitadores" (p. 42). La sociedad actual está inmersa en los avances tecnológicos, y los docentes e instituciones educativas deben dar cambios al enfoque pedagógico tradicional. Se hace necesario minimizar los efectos de la enseñanza con la tecnología sobre las emociones de los educandos, que germinan del "miedo infantil fruto del desconocimiento de la calidad de vida futura" (Martinez, 2018).

La tecnología está en todos los ámbitos de la vida cotidiana, lo cual permite que el educando esté conectado con una gran cantidad de información, donde, si esta no es controlada, curada y procesada produce consecuencias tal como el tecnoestrés. Por ende, el uso adecuado de la tecnología en el aula de clase de manera responsable tiene el beneficio de ayudar y elevar logros académicos de los estudiantes y las competencias digitales en los docentes.

\section{Desarrollo}

La generación de estudiantes del siglo XXI aprende de manera diferente. En el ámbito educativo el tema de las emociones no ha sido considerada por muchos años, pero, con la aparición de la Neurociencia que de acuerdo con el autor Salas (2003), es el "conjunto de ciencias cuyo sujeto de investigación es el sistema nervioso, con particular interés en cómo la actividad del cerebro se relaciona con la conducta y el aprendizaje" (p. 156), se puede reflexionar que como línea del pensamiento y acción trae como objetivo acercar a los agentes educativos a los conocimientos relacionados con el cerebro, aprendizaje, pedagogía y la psicología como necesarios para la innovación y transformación del fortalecimiento educativo.

Por lo tanto, es necesario que los docentes tengan presente que la construcción y reorganización de la actividad cognoscitiva está estrechamente ligada a las nuevas formas de experiencia social (Vygotsky, 1995), aspecto esencial de la calidad educativa. El 
desafío que existe actualmente en las aulas de clases es identificar cómo se sienten los educandos y docentes respecto a la práctica pedagógica apoyada en tecnología, para poder así reestructurar los currículos con base en su formación emocional y racional. Para lograr este objetivo se debe conocer cuáles son las emociones que acarrea utilizar la tecnología, para que de esa manera se facilite el control de las emociones.

Por lo antes expuesto, la presente investigación se basó en revelar el efecto de la tecnología en las emociones de los estudiantes, bajo un programa realizado desde el año 2013. La tecnología es el "conjunto de teorías y de técnicas que permiten el aprovechamiento práctico del conocimiento científico (Real Academia Española, 2007), por lo tanto, ha determinado la historia actual de la llamada sociedad del conocimiento y estructuran gran parte del tejido social; son muchos los expertos que han estudiado su impacto óptico, cognitivo y emocional sobre estas. La sociedad se mira a sí misma con un espejo tecnológico, que representa y entiende la realidad en la que se desarrolla a través de pantallas que traen hasta el espectador su propia imagen, conformando la experiencia visual de la sociedad actual.

Para poder reflexionar sobre la influencia emocional de la tecnología es necesario conocer qué es una emoción. Una emoción es define como "una experiencia corporal viva, veraz, situada y transitoria que impregna el flujo de conciencia de una persona" (Denzin, 2009, p. 66). Es decir, la transformación social producto del auge de las tecnologías, generan la necesidad de desarrollar competencias que permitan al individuo adaptarse con éxito a los cambios vertiginoso y a la sociedad, ya que los humanos se interesan por aquello que los motiva.

No obstante, son muchos los motivos, aunque algunos teóricos de la psicología apuntan como principal causa emocional de las aulas, la existencia de un modelo de organización escolar rígido, poco flexible, descontextualizado de la realidad socialeconómica actual y por el desface profesional de los docentes. Por lo tanto, la tecnología exige que los docentes desempeñen nuevas funciones" (Makrakis, 2005, p. 7) y entre ellas una formación sistemática que profundice el cómo aprender emocionalmente.

Desde luego, es tal la rapidez de instauración de la tecnología que se ha denominado a las personas nacidas a partir de los años 80, cómo la generación Net, que corresponde a los individuos nativos de las pantallas. En 2001, ocurrió la división planteada por el autor Prensky "los nativos digitales o aquellos que han nacido en un mundo ya digital, y los inmigrantes digitales personas que no han nacido en ese mundo" (p. 2), pero ellos deben adaptarse de una forma para aprenderlo sobre la tecnología.

Ahora bien, ante el bombardeo constante de la tecnología se corre el riesgo de caer en una actitud de indiferencia, llegando a una percepción fragmentada de la realidad e infravalorando la experiencia estética como experiencia del conocimiento. En este sentido, se puede afirmar que no todas las personas aceptan la tecnología, ni siquiera se plantean convertirse en inmigrantes digitales; incluso, dependiendo de las reacciones de los sujetos ante las Tecnologías del Aprendizaje y del Conocimiento (TAC), donde se hace una clasificación de cuatro categorías: Los entusiastas, los partidarios, los modernos primitivistas, y los laditas, que son los críticos de la tecnología que quieren acabar con la dependencia que actualmente tiene de ellas la humanidad.

En la actualidad, las TAC son un tema preocupante en diversas en áreas, incluso en el ámbito educativo, puesto que el impacto tecnológico no solo afecta a nivel personal y/o social, sino que puede ser un importante componente en el rendimiento laboral y emocional. Se debe tener presente que las emociones se utilizan para adecuar a la biósfera, son la expresión biológica que se refleja en el cuerpo y en la vida, tal como lo sustenta Zeballos (2020) "es fundamental el desarrollo profesional basado en el diagnóstico de las necesidades de formación" (p. 12).

En el año 1984, el autor Brod denomino tecnoestrés a la enfermedad causada por la falta de habilidad para adaptarse a las nuevas tecnologías de manera saludable, es decir, un trastorno de tipo adaptativo y motivado especialmente por una falta de autoeficacia para manejarse con las nuevas tecnologías, es decir, por una incompetencia ante las mismas. Por ello, si se parte del sentido biológico de la ansiedad: la supervivencia, la fatiga que parte del sentido biológico: cansancio emocional o agotamiento. Y el sentido biológico de la adicción parte de la necesidad consiente e inconsciente de la compañía para afrontar un reto. Ahora bien, es importante señalar cuales son los efectos emocionales de la tecnología durante la enseñanza, entre ellos tenemos de acuerdo con Instituto Nacional de Seguridad y Salud en el Trabajo (INSST) (2019): 
1. Tecnoansiedad: La persona percibe una fuerte tensión psicológica, egodistónica y malestar, ante el uso presente o posible de algún tipo de nuevas tecnologías. Esta tecnoansiedad, podría ser la entrada a la tecnofobia.

2. Tecnofatiga: La persona se caracteriza por sensaciones de cansancio y agotamiento mental y cognitivo, debido al uso de la tecnología, puede acompañarse también de actitudes recelosas y creencias de ineficacia. Un tipo específico de tecnofatiga sería el síndrome de fatiga informativa.

3. Tecnoadicción: Que sería un tecnoestrés específico, debido a un descontrol de los impulsos, mediante el cual el sujeto se vería obligado a utilizar TIC, a cualquier hora y en cualquier lugar, por tanto, durante largos periodos de tiempo (p. 2).

Desde la antigüedad al hombre le ha asustado la innovación, ha sentido temor ante la tecnología, un ejemplo sencillo de esto sería cuando los griegos no vinculaban las proezas técnicas e instrumentales con el progreso humano, pensaban que el uso de las máquinas podía llevar a provocar un cataclismo. En 1998 cuando algunos autores clasificaron a los usuarios de la tecnología, como normales, ansiosos y fóbicos, es decir los ansiosos: serían quienes se sienten incómodos al tener que utilizar los medios tecnológicos, pero no reportan síntomas físicos ni comportamientos de aversión frente a los mismos, mientras que los fóbicos: serían quienes evitan utilizar la tecnología porque tienen sentimientos de irrealidad, miedo a perder el control y refieren síntomas tales como palmas de las manos sudorosas, palpitaciones, temblores, entre otros. Y la tecnofilia sería el antónimo de tecnofobia, como la misma palabra expresa, se trata del apego a la tecnología, preexistiendo diferentes grados de tecnofilia dependiendo de la afinidad o dependencia que la persona tenga hacia la tecnología.

En el año 2010, los autores De la GándaraMartín y Álvarez definieron las cuatro adicciones más presentes en la tecnología y son:

5. Teleadicción, teledependencia: Es el rasgo principal es el uso excesivo o abusivo y pasivo de la $\mathrm{TV}$, con pérdida de la capacidad de resistirse a su uso; se hace presente el síndrome de abstinencia con irritabilidad, nerviosismo, insomnio, aturdimiento, cefaleas, entre otros.

1. Teleabuso: El patrón del uso exorbitante y frecuente que causa una especie de "intoxicación crónica" del TV.

2. Telefijación: El hábito extraño de observar la TV, que activa la absorción absoluta de la pantalla.

3. Telefonía móvil: Es un instrumento de comunicación, que beneficia e indemniza las relaciones sociales, por ello, su potencialidad adictógena es muy alta. Las personas exteriorizan irritabilidad, nerviosismo, insomnio, agresividad.

4. Videojuegos: La dependencia causa pérdida del control de los impulsos, lo que acarrea a un uso desenfrenado, síndrome de abstinencia y detención social (p. 12).

Por lo tanto, la tecnología, es una realidad de la cual no podemos escapar; se han hecho imprescindibles en la información, educación y en la comunicación. Realmente, los inmigrantes digitales tendrán que aprender por ensayo-error, pero a los nativos digitales se les puede educar y debe hacerse en el contexto que les ha tocado vivir, un mundo digitalizado y culturalmente lleno de imágenes. Por ende, la tarea pedagógica activa de los padres y docentes deberá acompañarse también de una enseñanza crítica en su crecimiento ascendente, para desarrollar adecuadamente su relación con el mundo cultural (digital). En este ámbito, es conveniente asegurar una pluralidad de recursos y de capacidades para afrontar los desafíos que pueden encontrar a lo largo de su desarrollo cognoscitivo, social, y físico.

El propósito de este estudio fue identificar las dificultades y problemas ocasionadas por las emociones, entre ellos: 1) conocer los conocimientos de los estudiantes-docentes sobre el uso de tecnología en las aulas de clases; y 2) describir el uso general de la tecnología en las aulas. El objetivo no fue solo comprender la necesidad y la importancia de la tecnología en las aulas en su relación con la motivación y el uso, sino revelar la realidad social desde una perspectiva objetiva, para así poder crear un plan de estudios basado en realimentación desde la Tecnoeducación, porque la tecnología no solo provee gestión pedagógica; sino que, además, de manera cíclica originan cultura informacional entre todos los 
involucrados del proceso de enseñanza-aprendizaje.

Los resultados del estudio lograron comunicar a una amplia audiencia a nivel internacional los beneficios e importancia y alineación del currículo basado en la motivación tecnológica. Lo hallazgos presentados fueron estudiados desde la siguiente interrogante: ¿Los docentes empelan la tecnología para motivar a los estudiantes-docentes a realizar las actividades escolares?

\section{Metodología}

En la actualidad, el sistema educativo se encuentra en una etapa de gran transformación. Está enfrentando los desafíos de la expansión, y para su reconstrucción todos los involucrados en el proceso de enseñanza-aprendizaje necesitan adoptar la tecnología y explorar los nuevos caminos para alcanzar la meta de calidad educativa. Como todos conocen, la educación tradicional es la que proporciona el componente humano que es muy necesario para el proceso de enseñanza-aprendizaje. Debido a que la personalidad y el comportamiento de los docentes influyen directamente en la personalidad de los estudiantes, la interacción cara a cara cumple con lo afectivo junto con los cognitivo y psicomotor, las habilidades sociales como cooperación, intercambio y expresión.

Desde lo antes mencionado, podemos afirmar que estamos en nueva era donde los estudiantes se han educado de manera diferente. El quehacer pedagógico debe cambiar en función de los tiempos actuales, se debe tomar en consideración las emociones en el aula e incluir en todos los niveles la enseñanza tecnoemocional. Para compensar el presente estudio se decidió proteger la identidad de los participantes, se utilizaron los datos obtenido de la plataforma Tecnológica-Educativa Docentes 2.0 que ayuda al docente tradicional que ha quedado desfasado con el avance de la tecnología. Este objetivo se realizó a través de un plan de estudios académico con énfasis en neurodidáctica.

El presente estudio fue una investigación de corte metodológico cuantitativo, el propósito de este fue revelar la realidad social desde una perspectiva objetiva para poder así crear planes pedagógicos para mejorar la instrucción Tecnológica-Educativa. Por lo tanto, las observaciones ayudaron a responder la pregunta de la investigación sobre si ¿Los docentes emplean la tecnología para motivar a los estudiantes- docentes a realizar las actividades escolares?

Se compilaron datos descriptivos de tres fuentes: Resultados de encuestas, registros de estudiantes y observaciones directas de las aulas. La población objeto de estudio fue de 369 estudiantes-docentes en el momento de investigación que de manera voluntaria realizó una encuesta anónima. Esta encuesta de siete (7) preguntas fue realizada durante el lapso académico enero-julio 2016 a través de la plataforma educativa.

Los resultados se compilaron en tablas digitales. La segunda fuente de información fueron los registros individuales de los estudiantes. Es pertinente señalar que en la documentación examinada e informada representó a todos los educandos de la plataforma, no a solo una muestra de la población. La última fuente de datos fueron las observaciones en el aula. El aula seleccionada fue Neurodidáctica con la intensión de tener un aula estándar donde el docente debe específicamente implementar adaptaciones para apoyar y cumplir los objetivos de estudios.

\section{Resultados}

Para cumplir con el propósito de este estudio, se tomaron decisiones para poder identificar el progreso de la implementación de la tecnología y sus efectos en un estudiante población de estudio, tales como la participación y observación del investigador en un plan de implementación de tecnología en la plataforma virtual. Durante este tiempo, se documentó todo el proceso de enseñanza-aprendizaje, luego se gestionó una encuesta para medir la motivación e interés de los estudiantes por la tecnología.

\section{Figura 1}

Edad de los encuestados.

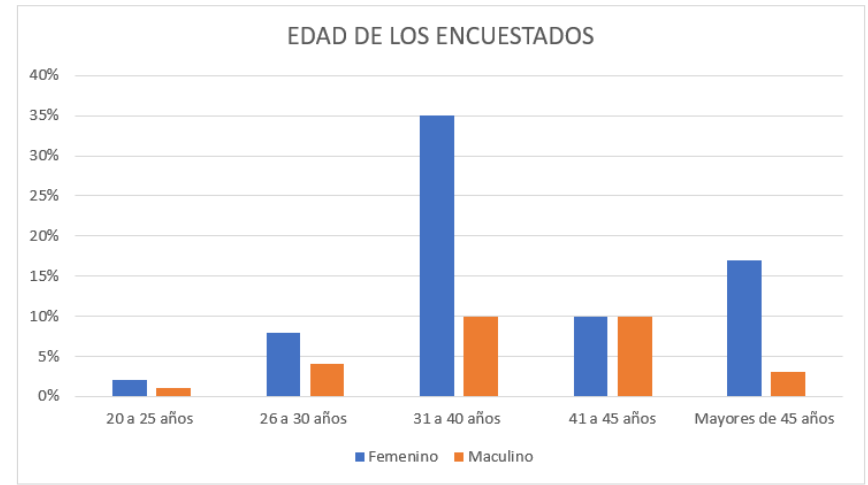

Nota. Encuesta para medir la motivación e interés de los estudiantes por la tecnología, elaboración propia. 
Se encuestaron un total de 369 estudiantesdocentes de la plataforma Tecnológica-Educativa Docentes 2.0 , de quienes el $72 \%$ fueron de sexo femenino y un $28 \%$ masculino. La edad de los encuestados se estableció a partir de los siguientes rangos: Las edades comprendidas entre 20 a 25 años (3\%), 26 a 30 años (12\%), 31 a 40 años (45\%), 41 a 45 años, (20\%) y mayores de 45 años $(20 \%)$.

\section{Figura 2}

El uso de la computadora en el hogar.

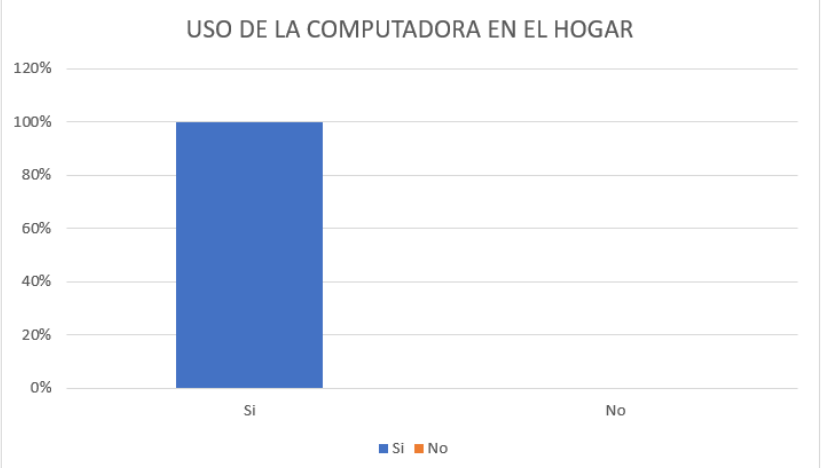

Nota. Diagnóstico del encuestado para determinar si posee herramientas tecnológicas en sus hogares, elaboración propia.

Se realizó una pregunta cerrada de sí o no, sobre si el encuestado tenían una herramienta tecnológica en sus hogares. Identificados como, computadora portátil, computadora de escritorio, tableta, teléfono o cualquier otro dispositivo que podría usarse para el trabajo relacionado con la instrucción pedagógica. De los 369 encuestados, 100\% respondieron "sí".

\section{Figura 3}

Los encuestados a la pregunta sobre el uso de la computadora para el quehacer pedagógico.

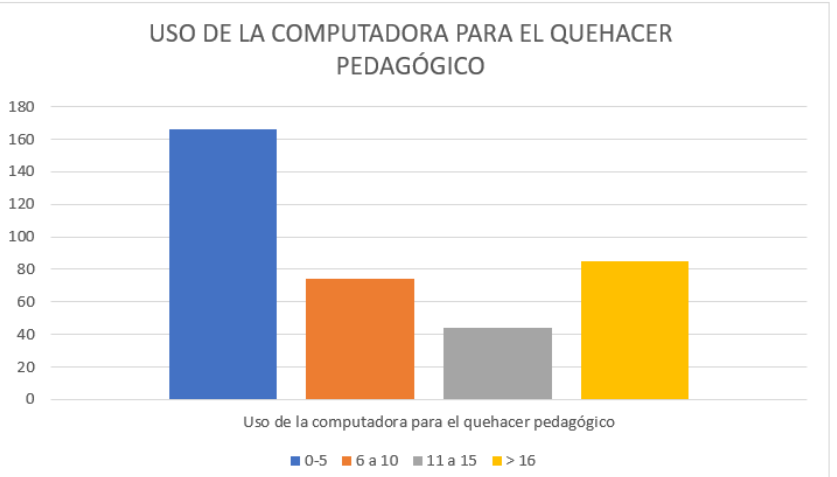

Nota. Diagnóstico sobre la frecuencia del uso de la computadora para el quehacer pedagógico o actividades conexas con el aula, elaboración propia.
La pregunta se realizó en opción múltiple. En relación con a la frecuencia del uso de la computadora para el quehacer pedagógico o actividades conexas con el aula. Los estudiantes tenían cuatro opciones para elegir. 166 estudiantes-docentes respondieron que usaban su computadora para actividades en el aula de 0 a 5 horas por semana, 74 declararon que usaban su computadora de 6 a 10 horas, 44 estudiantes declararon 11 a 15 horas y 85 estudiantes declararon 16 horas o más para el trabajo afín con el aula en una computadora en casa.

\section{Figura 4}

Los encuestados a la pregunta sobre la motivación a través de la tecnología.

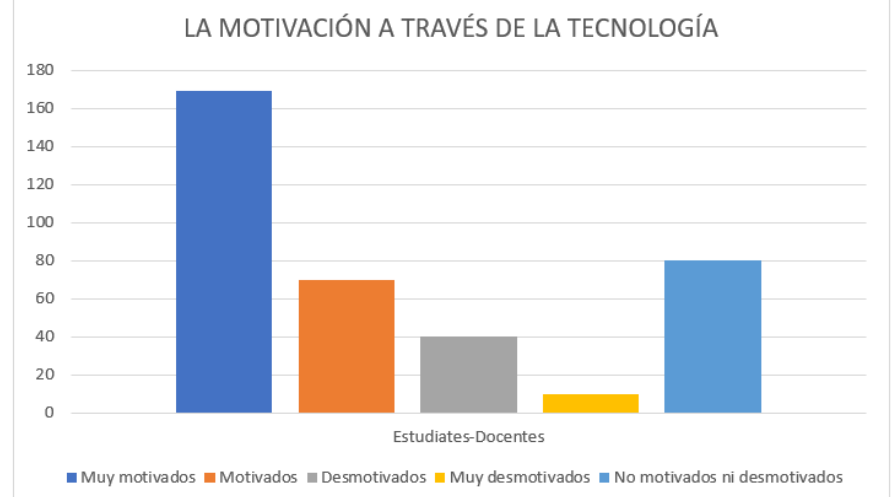

Nota. Elaboración propia.

Se elaboró una interrogante de opción múltiple, sobre qué tan motivado se siente el encuestado cuando utiliza la tecnología en el aula. De los 169 estudiantes-docentes declararon sentirse muy motivados, 70 declararon que solo se sentían motivados, 80 declararon que no se sentían motivados ni desmotivados, 40 estudiante declaró que se sentía desmotivado, y 10 encuestados respondieron muy desmotivados.

\section{Figura 5}

Los encuestados a la pregunta sobre el uso de la tecnología en clase.

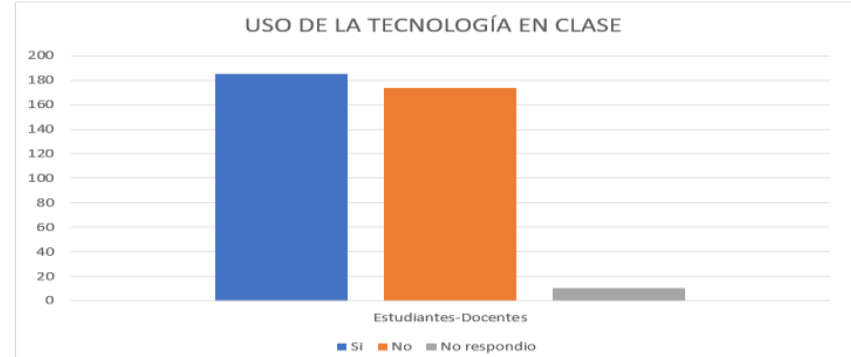

Nota. Diagnóstico sobre la utilización de la tecnología para el quehacer pedagógico, elaboración propia. 


\section{Conclusiones}

Debido a la progresiva importancia e incidencia del tecnoestrés en la educación actual, se puede hacer mención del estudio del autor Hudiburg (1996) quien realizó una investigación para indagar sobre las causas que establecían los problemas a los usuarios de la informática., lo cual dicho estudio generó la escala "Computer Hassles Scale" que mide el tecnoestrés.

Los resultados obtenidos en la presenta investigación arrojó que las causas de la desmotivación en los estudiantes fueron los siguientes problemas: Técnicos, de información, de falta de experiencia, de exigencias en cuanto al tiempo, los cuales son factores que contribuyen al tecnoestrés. Como medida preventiva al desarrollo de fatiga, ansiedad y adición es necesario la alfabetización digital de los docentes en las aulas virtuales. Los resultados manifiestan que la integración previa de la tecnología en que hacer pedagógico reduce los niveles de ansiedad. A partir de lo antes expuesto, se pudo observar una tendencia con las dimensiones del tecnoestrés entre las edades comprendidas de 31-40 años, esto podría corresponder a que son sus primeras prácticas en la docencia.

La tecnología, ha determinado la historia actual de la llamada sociedad del conocimiento y estructuran gran parte del tejido social; son muchos los expertos que han estudiado su impacto óptico, cognitivo y emocional. La sociedad se mira a sí misma con un espejo tecnológico, que representa y entiende la realidad en la que se desarrolla a través de pantallas que traen hasta el espectador su propia imagen, conformando la experiencia visual de la sociedad actual. La tecnología está en todos los ámbitos de la vida cotidiana, lo cual permite que el educando esté conectado con una gran cantidad de información. El uso adecuado de la tecnología en el aula ayuda a elevar los logros académicos de los estudiantes y las competencias digitales en los docentes, pero muchas instituciones educativas $\mathrm{y}$ docentes no han internalizado que ha llegado la educación digital para quedarse, un ejemplo palpable en tiempo de pandemia.

El cambio del paradigma tradicional ya está presente, y se sucedió bruscamente en un tiempo de pandemia, la implementación de la tecnología en la educación es imprescindible para asegurar que las instituciones educativas permanezcan y los quehaceres pedagógicos sustenten los aprendizajes significativos, ya que es el apoyo de las futuras carreras del mundo globalizado.

La ansiedad en tiempo de pandemia ha originado pensamientos de intranquilidad o miedo al futuro. Pero, el ser humano tiene mucha capacidad de resiliencia, seguro que usted encontrará la forma de salir de esta ansiedad. Lo trascendental es fijar la meta en la superación para no permanecer sumergido en el fracaso o en la queja, porque la ansiedad acrecentará. En el momento actual, se suman las tendencias mundiales del comportamiento social con intervención directa de la tecnología, lo cual, produjo una nueva visión de entender los ciclos comunes de la nueva sociedad, donde el ámbito de la salud, político, económico, institucional, como, además, el educativo a nivel mundial, se masificaron en este tiempo de pandemia para encontrar una nueva manera de describir el mundo: la globalización (Mann, 2004).

En síntesis, el reto es fortalecer al docente a nivel mundial. Intentando de que el conocimiento se correlacione con el presente avance tecnológico y la globalización, para poder así minimizar los errores de enseñanza, calidad, asimismo, aumentar exposición de estudiantes proceso de enseñanzaaprendizaje apoyado por las Tecnologías del Aprendizaje y del Conocimiento (TAC). La enseñanza apoyada por las TAC proporciona nuevas dimensiones del proceso de enseñanza-aprendizaje, y como sustenta Mujica-Sequera (2020) en el blog Docentes 2.0 "presenta a los estudiantes un amplio conjunto de conocimientos e innumerables oportunidades para aprender, desaprender y volver a aprender". Hoy en día, se pueden observar en las aulas de clases estudiantes desmotivados, es hora de gran reto educar desde la tecnoemoción.

\section{Referencias}

Barráez, D. (2020). La educación a distancia en los procesos educativos: Contribuye significativamente al aprendizaje. Revista Tecnológica-Educativa Docentes 2.0, 8(1), 41-49, https://ojs.docentes20.com/index.php/revistadocentes20/article/view/91

Brod, C. (1984). Technostress. Reading, Mass: Addison-Wesley.

De la Gándara-Martín, J.J., Álvarez Álvarez-Monteserin, M.T. (2010). Adicciones emergentes. En: Vallejo Ruiloba J, Leal Cercós C, Tratado de Psiquiatría Volumen I. $2^{\circ}$ edición (pp. 913-924). Barcelona: Ars Medica. 
Denzin, NK. (2009). On Understanding Emotion. New Brunswick, NJ: Transaction Publishers.

Hudiburg, RA. (1995). Psychology of computer use: XXXIV. The Computer Hassles Scale: subscales, norms, and reliability. DOI: 10.2466/pr0.1995.77.3.779

Instituto Nacional de Seguridad y Salud en el Trabajo (INSST) (2019). Tipo de tecnoestrés. Recuperado de https://www.insst.es/-/-quees-el-tecnoestres-

Makrakis (2005). Training teachers for new roles in the new era: Experiences from the United Arab Emirates ICT program, en Actas de la Tercera Conferencia Panhelénica sobre Didáctica de la Informática, Corinto (Grecia).

Mann, C. L. (2004). This is Bangaore calling: hang up. What technology. enable international trade in services U.S. Economy and Workforces. Federal Reserve Bank of Cleveland. Research Department. P.O. Ebsco- Academia- Search Premie

Martinez, M. (2018). ¿Te da miedo la tecnología? En su día, la electricidad dio miedo. Recuperado de https://www.nobbot.com/futuro/te-da-miedo-la-tecnologia/

Mujica-Sequera, R. (2018). Alfabetismo Digital. Recuperado de $\mathrm{https} / / /$ blog.docentes20.com/2018/12/alfabetizacion-digitaldocentes-2-0/

Mujica-Sequera, R. (2020). ¿Qué es Tecnoestrés? Recuperado de: https://blog.docentes20.com/2020/01/que-es-el-tecnoestresdocentes-2-0/

Prensky, M. (2001). Digital natives, digital immigrants' part 1. On the Horizon-The Strategic Planning Resource for Education Professionals n. 1 Vol. 6. Recuperado de http://www.ingentaconnect.com/content/mcb/274/2001/0000000 9/00000005/art00001

Real Academia Española. (2014). Diccionario de la lengua española (23.a ed.). Consultado en https://dle.rae.es/tecnolog\%C3\%ADa?m=form

Salas, R. (2003). ¿La educación necesita realmente de la neurociencia? Estudios Pedagógicos, n. 29, 2003, pp. 155-171. http://dx.doi.org/10.4067/S0718-07052003000100011

UNESCO (1998). La educación superior en el siglo XXI. Visión y acción. Documento de trabajo de la conferencia Mundial sobre la Educación Superior. Recuperado de: https://unesdoc.unesco.org/ark:/48223/pf0000116345_spa

Vygotski, L. S. (1995). Obras Escogidas. Visor. Madrid.

Zeballos, M. (2020). La evaluación de los aprendizajes mediadas por las TAC. Revista Tecnológica- Educativa Docentes 2.0, 9(1), 8395. https://doi.org/10.37843/rted.v9i1.98 